Artículos

\title{
Ansiedad en universitarios durante la pandemia de COVID-19: un estudio cuantitativo
}

\section{Anxiety in College Students during the COVID-19 Pandemic: A Quantitative Study}

\author{
Irma Leticia Chávez Márquez
}

Universidad Autónoma de Chihuahua

\section{Resumen}

La pandemia de COVID-19 ha generado cambios en la educación universitaria, lo cual puede derivar en alteraciones de tipo emocional, como la ansiedad. El objetivo de la investigación fue evaluar el nivel de ansiedad durante la pandemia de COVID-19 en estudiantes universitarios. Se realizó un estudio cuantitativo, utilizando un instrumento de medición (alfa de Cronbach de .90) para determinar el grado de ansiedad-estado en 1460 estudiantes universitarios del estado de Chihuahua en México. Para determinar el grado de ansiedad se utilizó la adaptación española del cuestionario de ansiedad estado-rasgo STAI (State-Trait Anxiety Inventory) en su subescala ansiedad-estado. Los resultados indican que el factor de mayor preocupación para los universitarios es el crecimiento de la pandemia (35.79 $\%)$. Los síntomas con mayor puntuación fueron sentirse tenso y preocupado; los que presentaron una mayor desviación estándar fueron sentirse oprimido, aturdido y alterado. Se ameritan futuras investigaciones que den seguimiento a los índices de ansiedad durante la evolución de la pandemia COVID-19.

Palabras clave: ansiedad, estudiantes universitarios, pandemia, COVID-19, estrés

Irma Leticia Chávez Márquez. Universidad Autónoma de Chihuahua

https://orcid.org/0000-0002-6494-951X

Autor para correspondencia: lettychavez7@gmail.com 


\begin{abstract}
The COVID-19 pandemic has generated changes in university education, which could lead to emotional disturbances, such as anxiety. The aim for this research was to measure the level of anxiety in a COVID-19 pandemic environment in university students. A quantitative study was carried out, using an instrument (Cronbach's alpha of $=.90)$ to determine the degree of anxiety in 1,460 university students from the state of Chihuahua in Mexico. To determine the degree of anxiety, the Spanish adaptation of the STAI (State-Trait Anxiety Inventory) was used in its anxiety-state subscale, Spanish version corresponding to IDARE Inventory. The main results indicate that the factor of greatest concern for university students is the growth of the pandemic (35.79\%). The symptoms with the highest scores in the level of anxiety detected were feeling tense and worried; those with the highest standard deviation were feeling oppressed, dazed, and upset. Future research is warranted to monitor anxiety rates during the evolution of the COVID19 pandemic.
\end{abstract}

Keywords: anxiety, college students, pandemic, COVID-19, stress

DOI https://doi.org/10.36793/psicumex.v11i1.420

Recibido 28 de Febrero de 2021

Aceptado 30 de Junio de 2021

Publicado 05 de Diciembre de 2021 


\section{Introducción}

El 11 de marzo de 2020, la Organización Mundial de la Salud (WHO) caracterizó al COVID-19 como una pandemia, lo que transformó todos los ámbitos de la vida. A raíz de la necesidad de seguir las recomendaciones preventivas ante el virus, se crearon situaciones con algún grado de alteración emocional, como puede ser la presencia de ansiedad. Aunado a esto, se han generado cambios inmediatos, pues debido a la pandemia varias instituciones están trabajando en el desarrollo de infraestructura confiable de apoyo e información requerida por el aumento de pacientes afectados por el COVID-19 (Reeves et al., 2020).

Han ocurrido muchas pandemias a través del tiempo, pero debido a factores como sobrepoblación, urbanización, cambios climáticos y aumento de los viajes internacionales, las epidemias y las pandemias virales siguen aumentando (Esparza, 2016). La pandemia del COVID-19 es una crisis de tipo global, que alcanza cada aspecto de la vida, desde lo íntimo hasta lo público (Meade, 2020), por lo que la afectación en la población puede ser evidente o no percibirse de manera consciente.

En el desarrollo del ser humano, la vida universitaria viene a ser un factor considerado primordial, que ejerce influencia en el estilo de vida, el cual si no es adecuado puede afectar la salud y un buen rendimiento académico (Jiménez y Ojeda, 2017). De cara a la pandemia se generaron nuevos métodos educativos en todos los niveles, ante esto, las redes sociales jugaron un papel primordial, incidiendo con un cambio de esquema de aprendizaje que puede generar estrés y/o ansiedad. El aprendizaje con base en las redes sociales es asíncrono, lo que permite al estudiante interactuar a su propio ritmo con el contenido educativo en cualquier momento o lugar (Thamman et al., 2020), sin embargo, esto provoca un cambio de rutinas e incertidumbre ante el desarrollo de su educación en el futuro, lo que puede generar diversos niveles de estrés y finalmente ansiedad. 
El paso por la universidad es etapa fundamental, pues influye directamente en el futuro profesional. Los cambios de tipo social, económico y psicológico enfrentados definen el desarrollo de los universitarios(Jiménez y Ojeda, 2017). La salud mental de gran cantidad de alumnos se ve amenazada por cambios muy rápidos, exigencias nuevas, competitividad y crisis económica, pero no siempre se les presta la atención debida, pudiendo derivar en ansiedad (Martínez-Otero, 2014). Los estresores ambientales generan en los estudiantes cifras alarmantes de estrés de tipo académico, lo que causa diversas reacciones (Jayasankara et al., 2018) de tipo físicas y/o psicológicas que deben ser atendidas oportunamente.

El estrés es una reacción emocional cada vez más frecuente, generada por factores de muy diverso origen, y los universitarios pueden experimentarlo aún sin percatarse de ello. Las expectativas académicas, tanto de índole interna como externa, pueden ser una fuente de estrés.. Lo anterior, lleva al planteamiento de estrategias de intervención que sean eficientes y adecuadas, que contribuyan a la comprensión de una fuente de estrés, como lo es el ambiente académico (Jayasankara et al., 2018). Junto con el estrés, otro desajuste emocional de gran frecuencia es la ansiedad, la cual puede ser transitoria o llegar a ser un rasgo de la personalidad. La ansiedad en los estudiantes puede desencadenarse por situaciones externas o bien por estímulos internos, que pueden ocasionar reacciones fisiológicas y de conducta (Reyes et al., 2017), las cuales no siempre son detectadas y atendidas por profesionales.

Las nuevas estrategias para continuar con las actividades escolares se basaron principalmente en el uso intensivo del internet. Sin embargo, existen regiones que no contaban con la infraestructura necesaria para una rápida implementación de dichas estrategias. El uso del internet, por parte de estudiantes universitarios en países en desarrollo, tiene un efecto en el rendimiento académico y la comunicación (Destiny y Onosahwo, 2018), pues al no contar con la tecnología necesaria para lograr los objetivos educativos se puede generar estrés y ansiedad. Empero, existen técnicas fáciles de implementar con 
evaluaciones formativas, las cuales pueden lograr que los estudiantes se sientan cómodos, fungiendo como un efecto mediador entre la ansiedad y el rendimiento educativo (Molin et al., 2019).

Las redes sociales han sido un excelente aliado durante la pandemia, pues cuentan con la capacidad de interactuar y construir contenido educativo, y permiten al conocimiento ser más accesible en comparación con las formas tradicionales de aprendizaje, como son las conferencias en persona o los libros de texto (Thamman et al., 2020). Sin embargo, los entornos educativos pueden implementar programas que disminuyan o mejoren el afrontamiento del estrés (Pascoe et al., 2020). No obstante, estos planteamientos corresponden a contextos previos a la pandemia, debido a los cambios que han sufrido los entornos educativos aún no se sabe con certeza cual será el curso por seguir en la enseñanza universitaria.

Los estudiantes universitarios pueden sufrir ansiedad como una reacción multifacética ante la amenaza del fracaso, así como ante la evaluación de su aprendizaje, resolución de problemas, entre otras situaciones (Cooper et al., 2018), como puede ser la presencia de la pandemia. Los estudiantes con trastorno de ansiedad exhiben una actitud pasiva en sus estudios, falta de interés en el aprendizaje, bajo rendimiento en los exámenes y mal desempeño en las tareas (Vitasari et al., 2010). Con base en lo anterior, se puede decir que los universitarios son un grupo de riesgo con relación a la ansiedad, debido a las exigencias de tipo psicológico, social, académico y un marcado estrés (Cardona-Arias et al., 2015), asimismo, estas exigencias pueden verse acentuadas por la presencia del COVID-19.

La ansiedad ha sido definida como una condición de tipo emocional y transitoria en el organismo, se caracteriza por sentimientos de tipo subjetivo, como la tensión y la aprensión (Del Rio et al., 2018). La ansiedad-estado, según Spielberger (citado en Ries et al., 2012, p.9), es un “estado emocional inmediato, modificable en el tiempo, caracterizado por una combinación única de sentimientos de tensión, aprensión y nerviosismo, pensamientos molestos y preocupaciones, junto a cambios psicológicos”. 
El concepto de ansiedad ha sido muy estudiado, sin embargo, en ocasiones se emplean como sinónimos la angustia, miedo, estrés, entre otros (Martínez-Otero, 2014). Del Río et al. (2018) mencionan que es un concepto complicado de definir, por lo que citan a Sierra et al. (2003), quienes indican que se puede confundir con estrés o angustia. El alto nivel de ansiedad hace que la vida normal de una persona sea difícil, interfiere en sus actividades y en su vida social (Vitasari et al., 2010).

La ansiedad puede ser considerada como una respuesta de adaptación normal de frente a lo que se considera una amenaza (estrés), ya que permite a una persona mejorar su desempeño, aun cuando en ocasiones esta respuesta puede no ser la adecuada, debido a que el estrés puede ser excesivo para los recursos con que cuenta el individuo (Castillo et al., 2016). Ante estas situaciones anormales, ya sean o no de tipo temporal, deberán adoptarse estrategias de afrontamiento. Éstas son respuestas que los seres humanos dan para solucionar diversos eventos o situaciones con los recursos con que cuente el individuo los cuales serán determinantes para la efectividad de tal afrontamiento (Gantiva et al., 2010). El afrontamiento puede tener dos funciones: "la regulación de las emociones estresantes y la modificación de la relación problemática entre la persona y el ambiente que causa el estrés", está orientado a lo que se piensa y se hace en situaciones generadoras de estrés, es contextual y podrá modificarse conforme se presenten los encuentros, pero será influenciado por la valoración personal del individuo en torno a una demanda real de lo que se enfrenta y con qué recursos se abordará (Cabanach et al., 2010, p.53).

En esta investigación se planteó como objetivo principal evaluar el nivel de ansiedad en un entorno de pandemia de COVID-19 en estudiantes universitarios, para lo cual se plantearon los siguientes objetivos específicos:

1. Determinar el factor que genera una mayor preocupación en los estudiantes durante la pandemia COVID-19. 
2. Establecer la relación entre las dimensiones de ansiedad estado-rasgo y el nivel de conocimiento sobre la pandemia de COVID-19.

3. Determinar el nivel de ansiedad presente en los estudiantes universitarios.

4. Delimitar las dimensiones del estado ansiedad estado-rasgo más predominantes en el nivel de ansiedad de los estudiantes.

\section{Metodología}

\section{Participantes}

Participaron 1476 estudiantes universitarios del estado de Chihuahua, México, en un momento en el cual los estudiantes universitarios se encuentran inscritos en una modalidad que se ofrece a distancia de manera virtual debido a la pandemia. Del total de participantes, $932(63.4 \%)$ fueron mujeres y $537(36.6 \%)$ fueron hombres; de entre18 y 25 años. El área de la carrera de los participantes correspondió a 643 (43.8 \%) de ciencias económico-administrativas, 431 (29.3\%) de ciencias de la salud, 236 (16.1\%) de ingeniería y química, $128(8.7 \%)$ de ciencias sociales y $31(2.1 \%)$ de ciencias agropecuarias.

\section{Instrumentos}

El cuestionario aplicado consistió en 31 preguntas cerradas y se dividió en 2 partes, la primera consistió en 11 preguntas con datos de la filiación del encuestado y sobre conocimiento e interés en información sobre el COVID-19. En la segunda parte del cuestionario se aplicó el cuestionario de ansiedad estado (STAI) con 20 preguntas divididas en 6 dimensiones (American Psychological Association, APA, 2011) con la adaptación al español que corresponde al inventario IDARE, el cual ha demostrado ser útil en la medición de ansiedad en estudiantes universitarios (Corsini et al., 2012).

Se utilizó el cuestionario de ansiedad estado-rasgo STAI (State-Trait Anxiety Inventory [STAI], Spielberger et al., 1970; Spielberger et al., 2008) compuesto por 40 ítems, diseñado para evaluar dos 
conceptos independientes de la ansiedad: la ansiedad como estado (condición emocional transitoria) y la ansiedad como rasgo (propensión ansiosa relativamente estable). Se conformó un marco de referencia para la ansiedad como estado (20 ítems) únicamente, ya que se buscó medir un nivel de ansiedad durante un evento inusual como lo es la pandemia COVID-19, pues la ansiedad como estado "evalúa un estado emocional transitorio, caracterizado por sentimientos subjetivos, conscientemente percibidos, de atención y aprensión y por hiperactividad del sistema nervioso autónomo" (Cabello et al., 2014, p.4). Para la escala, se utilizó un sistema de respuesta de Likert de 4 puntos, de acuerdo con la respuesta (1= casi nunca/nada, 2= algo/a veces, $3=$ con frecuencia/a menudo y $4=$ casi siempre/mucho). La puntuación total en la subescala ansiedad estado oscila entre 20 y 80 puntos (Fonseca-Pedrero et al., 2012). De acuerdo con Spielberg (1983, citado en Castillo et al., 2016, párr. 10), los totales de "ansiedad rasgo y de ansiedad estado abarcan desde 0 hasta 60 , y una mayor puntuación corresponde a un mayor grado de ansiedad. Puntuaciones $\geq 30$ sugieren ansiedad moderada y puntuaciones $\geq 45$ una ansiedad severa", por lo cual puntuaciones $\leq 29$ sugieren ansiedad baja.

En la subescala de ansiedad estado, se dividió en las siguientes dimensiones de acuerdo a la adaptación realizada por Burgos y Gutiérrez (2013): tranquilidad, aproblemado, bienestar, desconfort, preocupación y tensión, las cuales se describen a continuación.

\section{Dimensión tranquilidad}

Tranquilidad: la persona se percibe a sí misma en este momento con su cuerpo relajado, en calma, teniendo control de la situación. Se conformó por 8 ítems:

1. Me siento calmado.

2. Me siento seguro.

5. Me siento cómodo (estoy a gusto). 
10. Me siento confortable.

11. Tengo confianza en mí mismo.

15. Estoy relajado.

19. Me siento alegre.

20. En este momento me siento bien.

\section{Dimensión aproblemado}

Aproblemado: la persona se percibe a sí misma con muchas situaciones conflictivas (problemas) y que son difíciles de resolver de manera asertiva. Fue conformada por 4 ítems:

1. Estoy afligido.

12. Me siento nervioso.

17. Estoy preocupado.

18. Me siento aturdido (alterado).

\section{Dimensión bienestar}

Bienestar: estado en el cual una persona se siente satisfecha respecto a la situación en la que se encuentra. Se compuso de 2 ítems:

3. Estoy tenso.

16. Me siento satisfecho.

\section{Dimensión desconfort}

Desconfort: este factor corresponde a un estado con señales de alertas, las cuales generan un estado de inseguridad. Se incluyeron 2 ítems:

7. Estoy preocupado ahora por posibles desgracias futuras.

14. Me siento muy atado (como oprimido). 


\section{Dimensión preocupación}

Preocupación: corresponde a un estado emocional transitorio en donde la persona se encuentra alterada por una señal que puede ser identificable. Se conformó por 3 ítems:

6. Me siento alterado.

8. Me siento descansado.

9. Me siento angustiado.

\section{Dimensión tensión}

Tensión: estado en el cual el sujeto percibe su cuerpo rígido e intranquilo. Se midió con un ítem:

13. Estoy inquieto.

El nivel de ansiedad se obtiene restando el total de la puntuación de los ítems que reflejan calma (1, $2,5,8,10,11,15,16,19$ y 20) de la puntuación de los ítems que reflejan angustia $(3,4,6,7,9,12,13,14$, 17 y 18$)$.

\section{Procedimiento}

Para la presente investigación se contó con una muestra total de 1476 estudiantes universitarios, con un muestreo probabilístico aleatorio simple del total de la población inscrita, la cual consta de 126868 estudiantes inscritos en el estado de Chihuahua, México en educación superior (SEP, 2019). La investigación fue realizada en el periodo comprendido entre marzo y junio de 2020. El instrumento de medición se aplicó mediante un cuestionario en línea, el cual fue enviado por medio de correo electrónico. Los participantes manifestaron su consentimiento informado para el manejo de sus respuestas. 


\section{Análisis de datos}

En este trabajo, la ansiedad se trata de acuerdo al abordaje de ansiedad estado (AE). Spielberger et al. (1997, citado en Martínez-Otero, 2014) hacen una diferenciación entre ansiedad estado (AE) y ansiedad rasgo (AR); donde la ansiedad estado (AE) se considera como un estado o condición emocional transitoria en relación al tiempo y fluctúa su intensidad, la cual puede caracterizarse por sentimientos de tipo subjetivos, así como por la percepción de aprensión y tensión, además de una hiperactividad del sistema nervioso autónomo; mientras que la ansiedad rasgo (AR) es relativamente una propensión ansiosa de tipo estable en la persona.

El cuestionario aplicado de ansiedad estado (STAI) fue validado previamente con un valor alfa de Cronbach de 0.902 (Del Rio et al., 2018); en este estudio, para validar la consistencia del instrumento de medición aplicado, se obtuvo el coeficiente alfa de Cronbach, el cual dio un resultado aceptable (0.70), de acuerdo con Spielberg (1993) los coeficientes test-retest para esta medida pueden variar de 0.69 a 0.89 , además, se indica que un número considerable de pruebas atestiguan la construcción y la validez concurrente de la escala.

Se aplicó la prueba de adecuación muestral KMO (Kaiser-Meyer-Olkin), la cual arrojó un valor alto de 0.954 , con un valor $\mathrm{p}=0.0$, lo que respalda la correlación entre las variables consideradas para el procesamiento de datos con un análisis factorial de componentes principales. También se obtuvo la prueba de esfericidad de Bartlett, la cual arrojó un nivel de significancia (valor p) de 0.0, lo cual indicó que se puede aplicar el análisis factorial. Los análisis estadísticos se llevaron a cabo en el paquete estadístico SPSS versión 20.

Para el procesamiento de los datos, se fueron analizando de acuerdo con los objetivos planteados. Primero se determinó la percepción sobre el factor de mayor preocupación en un momento de pandemia de COVID-19. En segundo lugar, se procesaron los datos obtenidos mediante un análisis factorial para obtener 
componentes que indicaran la relación entre las dimensiones de ansiedad estado-rasgo y el conocimiento sobre la pandemia COVID-19. La tercera parte del procesamiento de los datos obtenidos consistió en la medición de niveles de ansiedad en los participantes de acuerdo con la siguiente escala para el nivel de ansiedad: bajo $\leq 29$, medio $=30$ a 44 y alto $\geq 45$ (Corsini et al., 2012; Castillo et al., 2016) para determinar el nivel de ansiedad presente en los estudiantes universitarios. Finalmente se delimitaron las dimensiones del estado-rasgo con mayor predominio en los estudiantes participantes en la investigación.

\section{Resultados}

A continuación, se presentan los resultados obtenidos con la aplicación del instrumento de medición.

\section{Percepción sobre factor de mayor preocupación durante la pandemia de COVID-19}

De acuerdo con la percepción de los estudiantes universitarios, el factor de mayor preocupación por la pandemia es el crecimiento de esta (36\%), seguido del detrimento de la economía (34 \%), y donde lo que les genera menor preocupación es la irregularidad en su situación escolar (30\%), como se puede observar en la figura 1. 


\section{Figura 1}

Percepción sobre mayor preocupación durante la pandemia

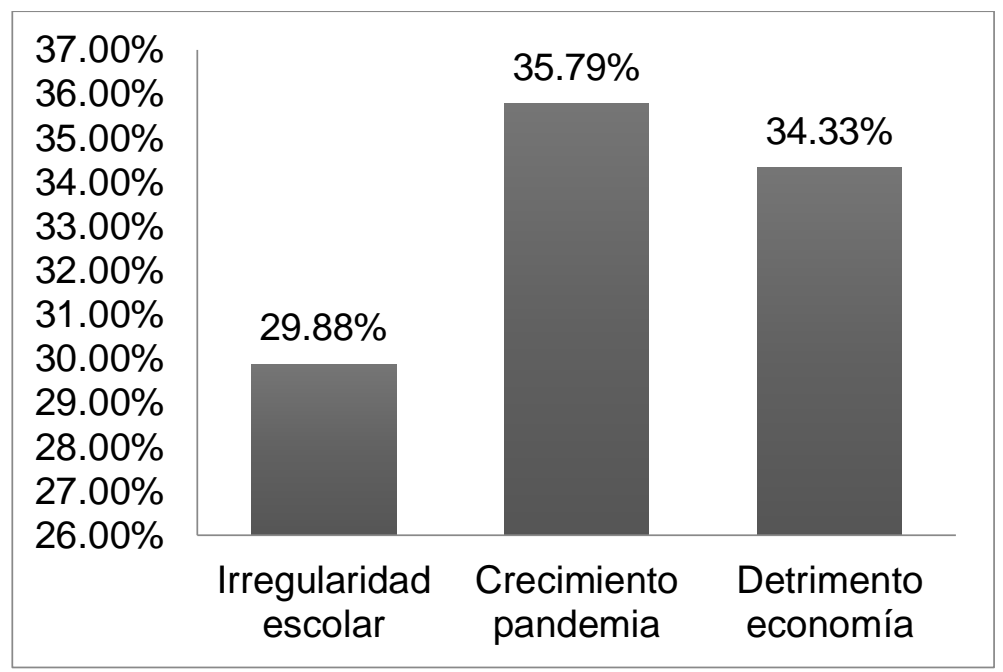

Fuente: elaboración propia

\section{Relación entre las dimensiones de ansiedad estado-rasgo y conocimiento sobre COVID-19}

Se extrajeron cuatro componentes mediante un análisis factorial, utilizando el método de extracción de componentes principales y la rotación varimax, donde previamente se determinó que el $53.85 \%$ de la varianza total se explicaba por cuatro componentes, donde el $24.43 \%$ de la varianza se explicó por el primer componente. En la tabla 1, se puede observar que los primeros 11 ítems explicaban el componente uno, el cual correspondería a manifestaciones de ansiedad según el cuestionario STAI (10) y la percepción del encuestado sobre sufrir estrés o ansiedad (1); el componente dos (19.93\% varianza) se explicó por 10 ítems correspondientes a la ausencia de ansiedad de acuerdo con el cuestionario STAI; el componente tres se explicó por tres ítems correspondientes al interés personal sobre el COVID-19 por diversas razones; finalmente, el componente cuatro es explicado únicamente por el medio por el cual se informa sobre el COVID-19 (ver tabla 1). 


\section{Tabla 1}

Matriz de componentes principales de cuestionario STAI y conocimiento, comportamiento e información en relación con el COVID-19

\begin{tabular}{|c|c|c|c|c|}
\hline & 1 & 2 & 3 & 4 \\
\hline Me siento angustiado & 0.746 & -0.287 & 0.022 & 0.001 \\
\hline Estoy tenso & 0.746 & -0.245 & -0.012 & 0.022 \\
\hline Me siento alterado & 0.745 & -0.272 & -0.034 & 0.058 \\
\hline Me siento nervioso & 0.729 & -0.243 & 0.034 & -0.003 \\
\hline Estoy preocupado & 0.722 & -0.233 & 0.136 & -0.039 \\
\hline Estoy afligido & 0.699 & -0.242 & -0.026 & 0.037 \\
\hline \multicolumn{5}{|l|}{ Me siento aturdido y } \\
\hline alterado & 0.679 & -0.287 & -0.014 & 0.122 \\
\hline Estoy inquieto & 0.677 & -0.241 & -0.045 & 0.128 \\
\hline Preocupado por el futuro & 0.639 & -0.125 & 0.135 & -0.108 \\
\hline Me siento oprimido & 0.579 & -0.319 & -0.091 & 0.189 \\
\hline \multicolumn{5}{|l|}{ Estrés, preocupación o } \\
\hline ansiedad & 0.519 & -0.215 & 0.119 & -0.142 \\
\hline Me siento satisfecho & -0.246 & 0.755 & 0.003 & -0.097 \\
\hline Me siento alegre & -0.229 & 0.748 & 0.03 & -0.077 \\
\hline Me siento bien & -0.263 & 0.743 & 0.086 & -0.021 \\
\hline Me siento cómodo & -0.286 & 0.71 & 0.01 & -0.021 \\
\hline
\end{tabular}


Tabla 1 (continuación)

\begin{tabular}{|c|c|c|c|c|}
\hline & 1 & 2 & 3 & 4 \\
\hline Me siento confortable & -0.213 & 0.708 & 0.05 & 0.05 \\
\hline Estoy relajado & -0.388 & 0.677 & -0.029 & -0.066 \\
\hline Me siento descansado & -0.152 & 0.607 & -0.006 & -0.042 \\
\hline Confianza en mí mismo & -0.218 & 0.583 & 0.127 & 0.162 \\
\hline Me siento seguro & -0.376 & 0.572 & 0.019 & 0.183 \\
\hline Me siento calmado & -0.49 & 0.552 & 0.021 & 0.136 \\
\hline \multicolumn{5}{|l|}{ Pendiente de } \\
\hline \multicolumn{5}{|l|}{ información sobre } \\
\hline COVID-19 & 0.058 & 0.053 & 0.801 & -0.072 \\
\hline \multicolumn{5}{|l|}{ Conocimiento sobre } \\
\hline COVID-19 & 0.017 & 0.009 & 0.687 & 0.06 \\
\hline \multicolumn{5}{|l|}{ Seguir recomendaciones } \\
\hline \multicolumn{5}{|l|}{ oficiales durante } \\
\hline pandemia COVID-19 & 0.067 & 0.071 & 0.525 & 0.097 \\
\hline \multicolumn{5}{|l|}{ Medio de información } \\
\hline sobre COVID-19 & -0.111 & 0.015 & 0.4 & 0.524 \\
\hline$\%$ Varianza & 23.431 & 19.939 & 6.318 & 4.167 \\
\hline
\end{tabular}

Fuente: elaboración propia 


\section{Medición de niveles de ansiedad en estudiantes}

Una vez obtenida la puntuación de los ítems de las dimensiones de ansiedad medida por medio del STAI, se procedió a obtener los niveles de ansiedad presentados en cada uno los participantes en la muestra de acuerdo con la siguiente escala para el nivel de ansiedad: bajo $=\leq 29$, medio $=30-44$ y alto $=45$ o más (Corsini et al., 2012; Castillo et al., 2016). Las puntuaciones obtenidas para nivel de ansiedad arrojaron que $469(31.77 \%)$ del total de 1476 estudiantes universitarios, presentan un nivel de ansiedad clasificado como bajo, con un valor medio; no obteniendo valores para niveles de ansiedad clasificados como medio y alto. No se encontraron diferencias significativas en cuanto a sexo, edad y área de la carrera en la cual están inscritos. Lo anterior se presenta en la tabla 2.

\section{Tabla 2}

Características de los universitarios y nivel de ansiedad-estado

\begin{tabular}{|c|c|c|c|c|}
\hline & Variable & $\begin{array}{c}\text { Frecuencia } \\
\text { (universitarios } \\
\text { con ansiedad) }\end{array}$ & $\begin{array}{c}\text { Porcentaje } \\
\text { (universitarios } \\
\text { con ansiedad) }\end{array}$ & $\begin{array}{c}\text { Porcentaje } \\
\text { (total de la } \\
\text { muestra) }\end{array}$ \\
\hline \multicolumn{5}{|l|}{ Sexo } \\
\hline & Mujer & 361 & 76.97 & 63.4 \\
\hline \multicolumn{5}{|l|}{$\begin{array}{l}\text { Área de la } \\
\text { carrera }\end{array}$} \\
\hline & $\begin{array}{l}\text { Económico- } \\
\text { administrativa }\end{array}$ & 184 & 39.23 & 43.8 \\
\hline & Salud & 126 & 26.86 & 29.3 \\
\hline & Ingeniería/Química & 93 & 19.82 & 16.1 \\
\hline & Ciencias Sociales & 52 & 11.08 & 8.7 \\
\hline & $\begin{array}{l}\text { Agropecuarias } \\
\text { Edad } \\
(\text { media }+/-D E)\end{array}$ & $\begin{array}{l}14 \\
20.5+/-2.1\end{array}$ & 2.98 & 2.1 \\
\hline \multirow{4}{*}{$\begin{array}{l}\text { Nivel } \\
\text { Ansiedad } \\
\text { Estado }\end{array}$} & Bajo $(\leq 29)$ & 469 & 100 & 31.77 \\
\hline & $\begin{array}{l}\text { Nivel ansiedad } \\
(\text { media }+/-D E)\end{array}$ & $8.35+/-6.02$ & & \\
\hline & Medio (30 a 44) & 0 & 0 & 0 \\
\hline & $\begin{array}{c}\text { Alto }(\geq 45) \\
\text { Edad } \\
(\text { media }+/-D E)\end{array}$ & $\begin{array}{l}0 \\
20.8+/-1.9\end{array}$ & 0 & 0 \\
\hline
\end{tabular}

Fuente: elaboración propia. 


\section{Dimensiones de la ansiedad estado-rasgo con mayor predominio en el nivel de ansiedad}

Para la determinación del nivel de ansiedad detectado, se obtuvo una media de 8.34 y una desviación típica de 6.02. Al delimitar las dimensiones con mayor predominio en los resultados obtenidos para la medición de ansiedad, con un mayor valor en la media aparecen "estoy tenso"(ítem 3, dimensión bienestar), “preocupado por el futuro"(ítem 7, dimensión desconfort) y “estoy preocupado” (ítem 17, dimensión aproblemado); mientras que los que presentan una mayor desviación típica son "me siento oprimido"(ítem 14, dimensión desconfort) y "me siento aturdido y alterado”(ítem 18, dimensión aproblemado), lo cual se puede observar en la tabla 3.

\section{Tabla 3}

Estadísticos descriptivos del nivel de ansiedad obtenido en estudiantes universitarios

\begin{tabular}{|c|c|c|c|c|}
\hline & $\begin{array}{c}\text { Valor } \\
\text { Mínimo }\end{array}$ & $\begin{array}{c}\text { Valor } \\
\text { Máximo }\end{array}$ & Media & $D E$ \\
\hline Nivel de ansiedad & 1 & 29 & 8.3497 & 6.02641 \\
\hline Me siento calmado & 1 & 4 & 2.0064 & 0.64547 \\
\hline Me siento seguro & 1 & 4 & 2.1258 & 0.68808 \\
\hline Estoy tenso & 1 & 4 & 3.0043 & 0.73959 \\
\hline Estoy afligido & 1 & 4 & 2.5906 & 0.79178 \\
\hline Me siento cómodo & 1 & 4 & 1.9531 & 0.62184 \\
\hline Me siento alterado & 1 & 4 & 2.597 & 0.79909 \\
\hline Preocupado por el futuro & 1 & 4 & 3.194 & 0.74728 \\
\hline Me siento descansado & 1 & 4 & 1.8507 & 0.74758 \\
\hline Me siento angustiado & 1 & 4 & 2.8827 & 0.7375 \\
\hline Me siento confortable & 1 & 4 & 1.8486 & 0.57411 \\
\hline Confianza en mí mismo & 1 & 4 & 2.4222 & 0.84024 \\
\hline Me siento nervioso & 1 & 4 & 2.8209 & 0.78304 \\
\hline Estoy inquieto & 1 & 4 & 2.951 & 0.80313 \\
\hline Me siento oprimido & 1 & 4 & 2.8358 & $\mathbf{0 . 8 8 4 7 7}$ \\
\hline Estoy relajado & 1 & 4 & 1.7441 & 0.5943 \\
\hline Me siento satisfecho & 1 & 4 & 1.7527 & 0.62925 \\
\hline Estoy preocupado & 1 & 4 & 3.1066 & 0.74057 \\
\hline $\begin{array}{l}\text { Me siento aturdido y } \\
\text { alterado }\end{array}$ & 1 & 4 & 2.5416 & 0.8554 \\
\hline
\end{tabular}




\section{Tabla 3 (continuación)}

\begin{tabular}{lcccc}
\hline Me siento alegre & 1 & 4 & 2.1258 & 0.68184 \\
Me siento bien & 1 & 4 & 2.3454 & 0.73966 \\
Total A (angustia) & 16 & 40 & 28.5245 & 4.6646 \\
Total B (calma) & 10 & 33 & 20.1748 & 3.42803 \\
\hline
\end{tabular}

Fuente: elaboración propia.

\section{Discusión}

Los estudiantes universitarios son un sector de la población que ha sido impactado gravemente por la pandemia del COVID-19, dado que no solo la situación irregular en su entorno los afecta —además del riesgo de salud que la misma situación de emergencia implica —, sino que su dinámica escolar ha cambiado en muchos sentidos, sobre todo para quienes se encuentran en la modalidad escolarizada y reciben su educación a distancia. Los estudiantes universitarios enfrentan retos de manera permanente durante su preparación académica, por lo cual estos, al igual que los adolescentes, son particularmente vulnerables a los problemas asociados con el estrés académico, el cual puede verse acentuado por condiciones anormales en su educación debido a la presencia de la pandemia, ya que las transiciones ocurren a nivel individual y social (Jayasankara et al., 2018).

Sin embargo, en esta investigación los estudiantes reportaron como factor de mayor preocupación el crecimiento de la pandemia, seguido del detrimento en la economía y en menor término la irregularidad en su situación escolar, la cual ha pasado de ser presencial a una modalidad virtual, a lo cual —a pesar de ser considerados nativos digitales - no estaban habituados en su gran mayoría; esto permite visualizar como han sido afectados por la emergencia sanitaria, pues esta puede ser el principal factor motivador de estrés, desplazando en mayor o menor medida al estrés académico. Ya previamente, respecto a la pandemia de H1N1 de 2009, Amela et al. (2010) habían expresado que si bien un cierre es benéfico para la salud, se generan costos económicos y sociales, pues los alumnos son afectados, lo cual puede provocar situaciones de angustia que generen ansiedad. El hecho de que su situación escolar no sea el factor que les genera mayor 
preocupación, puede deberse a que tienen un habitual manejo de la tecnología y el internet como parte de su vida diaria, como lo expresan Destiny y Onosahwo (2018), quienes indican que la utilidad percibida incide de forma significativa en la aceptación del manejo de dispositivos tecnológicos como el internet, ya que mejorarán su desempeño académico, esto indica que no necesariamente la educación a distancia sea lo que les genera altos niveles de angustia y ansiedad.

Aproximadamente la tercera parte $(31.77 \%)$ de los estudiantes presentaron ansiedad, sin embargo, todos ellos dentro del rango bajo de ansiedad-estado. Este porcentaje de estudiantes con ansiedad es muy similar al encontrado por Kamberi et al. (2019), quienes obtuvieron resultados que indicaban que la prevalencia de síntomas de ansiedad en una muestra de 676 estudiantes universitarios fue del $33.6 \%$. Mientras que Singh y Jha (2013) obtuvieron que un $46 \%$ de los estudiantes en una universidad Chilena tenían un alto nivel de ansiedad, sin estar ligado a una influencia de género, coincidiendo con los resultados obtenidos en esta investigación, donde se encontró que la ansiedad no estaba relacionada con ningún dato de filiación del estudiante, como la edad y el género, así como tampoco con el grado de conocimiento e información sobre COVID-19. Sin embargo, Kamberi et al. (2019) informaron de diferencias estadísticamente significativas en los síntomas de ansiedad por género, con una mayor prevalencia entre las mujeres. Pero, así como la ansiedad puede diferir según el individuo, también difiere entre subconjuntos demográficos de estudiantes en entornos académicos (England et al., 2019). Con base en lo expuesto, cabe resaltar que la investigación fue desarrollada con estudiantes que en su mayoría tienen acceso al internet, lo cual no es un factor motivador de estrés para ellos.

Entre quienes presentaron un nivel de ansiedad-estado, se encontró que es predominante el sentirse oprimido, así como sentirse aturdido y alterado; también quienes se sienten preocupados por el futuro manifiestan estar también angustiados, además, quienes manifestaron estar tensos, también están afligidos. 
Ya previamente se había indicado que algunos de los problemas observados en estudiantes con un alto estrés académico son la ansiedad, la depresión y problemas en relación al comportamiento (Deb et al., 2015).

El hecho de informarse acerca de la pandemia está altamente relacionado con el conocimiento sobre COVID-19. Como lo indica Casero-Ripollés (2020), ha resurgido el papel de los medios tradicionales, especialmente la televisión, ya que manifiesta una reconexión, por medio de las noticias, con los ciudadanos que generalmente permanecen lejos de la información, debido a que en este momento se difunden recomendaciones en constante evolución en lo referente al COVID-19 (Reeves et al., 2020), además de la información que fluye de manera constante en las redes sociales, que son un medio de comunicación con gran impacto en los universitarios.

Se ha demostrado que la ansiedad interfiere con el bienestar general de las personas, por lo cual su detección temprana se considera esencial para determinar intervenciones y programas necesarios; de acuerdo con la Estrategia 2020 de la Organización Mundial de la Salud en 2013, el fortalecimiento de los programas de promoción de la salud mental es sumamente relevante, pero se requieren estudios en profundidad para examinar otros factores relacionados con la ansiedad (Kamberi et al., 2019). No obstante, de acuerdo con Cabeza et al. (2018), cuando la ansiedad se mantiene en niveles estables y normalizados es benéfica, pues ayudará al afrontamiento de diversas situaciones propias de la vida en la universidad; por lo que se puede decir que la ansiedad cumple con una función de adaptación en el ser humano.

\section{Conclusiones}

Se concluye que los estudiantes universitarios han sido afectados por la presencia del COVID-19, tanto en su salud emocional como en la dinámica escolar generada. Contrario a lo esperado, su mayor preocupación es el crecimiento de la pandemia, y no su situación escolar. 
El principal resultado indica que aproximadamente un tercio de los estudiantes universitarios presentan ansiedad-estado en un nivel bajo. Sin embargo, ligado a ello, manifiestan sentirse oprimidos, aturdidos y alterados, lo cual genera angustia con un consecuente desequilibrio emocional.

Los resultados obtenidos en esta investigación dan pie a la realización de futuras investigaciones, que den seguimiento a los resultados obtenidos en relación con la manifestación de ansiedad, dado que este estudio fue realizado al inicio de la pandemia de COVID-19 en México, por lo que es importante determinar si se mantuvieron los mismos niveles de ansiedad o bien cambiaron al prolongarse la pandemia. Es de gran importancia la detección oportuna de la ansiedad, así como de otros problemas de tipo mental o emocional. Debido a que la evolución de la pandemia ha tenido un crecimiento constante en México, esta podría acentuar los niveles de ansiedad de los alumnos, en particular porque no existe certidumbre sobre el inicio de clases presenciales para las universidades, por lo que se recomienda a las autoridades educativas poner especial atención en la salud mental de los estudiantes universitarios.

Una de las principales limitaciones de esta investigación fue la pandemia de COVID-19, pues no permitió aplicar el instrumento de medición de manera presencial; por eso es importante continuar con futuros estudios de la mano de la evolución de la pandemia y su esperada estabilización, para descubrir si la ansiedad-estado cambia.

\section{Referencias}

Amela Heras, C., Cortes García, M. y Sierra Mores, M. J. (2010). Bases epidemiológicas para la toma de decisiones sobre medidas de salud pública (no farmacológicas) durante la respuesta a la pandemia de gripe (H1N1) 2009. Revista Española de Salud Pública, 84(5), 497-506. http://scielo.isciii.es/pdf/resp/v84n5/colaboracion3.pdf

American Psychological Association (APA). (2011). The State-Trait Anxiety Inventory (STAI). https://www.apa.org/pi/about/publications/caregivers/practice-settings/assessment/tools/trait-state 
Burgos Fonseca, P. y Gutiérrez Sepúlveda, A. (2013). Adaptación y Validación del Inventario Ansiedad Estado-Rasgo (STAI) en Población Universitaria de la Provincia de Ñuble. [Tesis de Licenciatura en Psicología, Universidad del Bío-Bío (Chile)]. http://repobib.ubiobio.cl/jspui/bitstream/123456789/265/3/Burgos\%20Fonseca_Pia.pdf

Cabanach, R. G., Valle, A., Rodríguez, S., Piñeiro, I. y Freire, C. (2010). Escala de afrontamiento del estrés académico (A-CEA). Revista Iberoamericana de Psicología y Salud, 1(1), 51-64. https://www.redalyc.org/pdf/2451/245116411005.pdf

Cabello Fernández, A., Franch Almela, P., Cabanes Hernández, E., Arnaus Paradís, T., Cabañas Carrasco M. y Martínez Vinuesa M. (2014). Aplicación de la encuesta de ansiedad STAI (state-trait ansiety inventory) para valorar la necesidad de sedación en pacientes de cribado de cáncer colorrectal. Enferm Endosc Dig., 1(2), 3-5. https://aeeed.com/documentos/publicos/revista/octubre2014/Enferm\%20Endosc\%20Dig.\%202014;1( 2)3-5.pdf

Cabeza Palacios, Á. E., Llumiquinga Guerrero, J. R., Vaca García, M. R. y Capote Lavandero, G. (2018). Niveles de ansiedad entre estudiantes que inician y culminan la carrera en actividad física. Revista Cubana de Investigaciones Biomédicas, 37(2), 54-62. http://scielo.sld.cu/scielo.php?script=sci_arttext\&pid=S0864-03002018000200007

Castillo Pimienta, C., Chacón de la Cruz, T. y Díaz-Véliz, G. (2016). Ansiedad y fuentes de estrés académico en estudiantes de carreras de la salud. Investigación en Educación Médica, 5(20), 230237. http://dx.doi.org/10.1016/j.riem.2016.03.001

Casero-Ripollés, A. (2020). Impact of Covid-19 on the Media System. Communicative and Democratic Consequences of News Consumption during the Outbreak. EPI, 29(2), 1-11. https://doi.org/10.3145/epi.2020.mar.23 
Cooper, K. M., Downing, V. R. y Brownell, S. E. (2018). The Influence of Active Learning Practices on Student Anxiety in Large-enrollment College Science Classrooms. International Journal of STEM Education, 5(23), 1-18. https://doi.org/10.1186/s40594-018-0123-6

Corsini M., G., Bustos M., L., Fuentes N., J. y Cantín, M. (2012). Niveles de Ansiedad en la Comunidad Estudiantil Odontológica. Universidad de La Frontera, Temuco - Chile. Int. J. Odontostomat, 6(1), 51-57. https://scielo.conicyt.cl/pdf/ijodontos/v6n1/art07.pdf

Deb, S., Strodl, E. y Sun, J. (2015). Academic Stress, Parental Pressure, Anxiety and Mental Health among Indian High School Students. International Journal of Psychology and Behavioral Sciences, 5(1), 26-34. https://doi:10.5923/j.ijpbs.20150501.04

Del Rio Olvera, F. J., Cabello Santamaría, F., Cabello García, M. A. y Aragón Vela, J. (2018).

Cuestionario de Ansiedad Estado Rasgo (STAI): análisis psicométrico y funcionamiento en una muestra de drogodependientes y controles. Universitas Psychologica, 17(1), 1-10. https://www.redalyc.org/jatsRepo/647/64754595008/64754595008.pdf

Destiny Apuke, O. y Onosahwo Iyendo, T. (2018). University Students' Usage of the Internet Resources for Research and Learning: Forms of Access and Perceptions of Utility. Heliyon, ELSEVIER, 4, 134. https://doi.org/10.1016/j.heliyon.2018.e01052

England, B. J., Brigati, J. R., Schussier, E. E. y Chen, M. M. (2019). Student Anxiety and Perception of Difficulty Impact Performance and Persistence in Introductory Biology Courses. CBE_Life Sciences Education, 18(21), 1-13. https://doi.org/10.1187/cbe.17-12-0284

Esparza, J. (2016). Editorial. Epidemias y pandemias virales emergentes: ¿Cuál será la próxima?. Investigación Clínica, 57(3), 231-235. https://www.redalyc.org/pdf/3729/372946635001.pdf 
Fonseca-Pedrero, E., Paino, M., Sierra-Braigrie, S., Lemos-Giráldez, S. y Muñiz, J. (2012). Propiedades psicométricas del "Cuestionario de Ansiedad Estado-Rasgo" (STAI) en universitarios. Behavioral Psychology / Psicología Conductual, 20(3), 547-561.

http://www.p3-info.es/PDF/PropiedadespsicomeetricasdelSTAI.pdf

Gantiva Díaz, C. A., Luna Viveros, A., Dávila, A. M. y Salgado, M. J. (2010). Estrategias de afrontamiento en personas con ansiedad. Psychologia. Avances de la disciplina, 4(1), 63-72. https://www.redalyc.org/pdf/2972/297224086006.pdf

Gobierno de México-Secretaría de Educación Pública (SEP). (2019). Principales cifras del sistema educativo nacional 2018-2019, México, SEP. https://www.planeacion.sep.gob.mx/Doc/estadistica_e_indicadores/principales_cifras/principales_cif ras_2018_2019_bolsillo.pdf

Jayasankara Reddy, K., Rajan Menon, K. y Thattil, A. (2018). Academic Stress and its Sources among University Students. Biomedical \& Pharmacology Journal, 11(1), 531-537. https://dx.doi.org/10.13005/bpj/1404

Jiménez Diez, O. y Ojeda López, R. (2017). Estudiantes universitarios y el estilo de vida. Revista Iberoamericana de Producción Académica y Gestión Educativa, 4(8). https://www.pag.org.mx/index.php/PAG/article/view/723

Kamberi, M., Hoxha, F., Shala, M., Shahini, M. y Vehapi, S. (2019). Anxiety Predictors among College Students in Kosovo. International Journal of Adolescence and Youth, 24(1), 117-124. https://doi.org/10.1080/02673843.2018.1479277

Martínez-Otero Pérez, V. (2014). Ansiedad en estudiantes universitarios: estudio de una muestra de alumnos de la Facultad de Educación. International Journal of Developmental and Educational Psychology, 1(1), 439-449. https://www.redalyc.org/pdf/3498/349851780045.pdf 
Meade, R. R. (2020). CDJ Editorial— What is this Covid-19 Crisis?. Community Development Journal, 55(3). https://doi.org/10.1093/cdj/bsaa013

Molin, F.; Cabus, S., Haelermans, C. y Groot, W. (2019). Toward Reducing Anxiety and Increasing Performance in Physics Education: Evidence from a Randomized Experiment. Research in Science Education. https://doi.org/10.1007/s11165-019-9845-9

Pascoe, M. C., Hetrick, S. E. y Parker, A. G. (2020). The Impact of Stress on Students in Secondary School and Higher Education. International Journal of Adolescence and Youth, 25(1), 104-112. https://doi.org/10.1080/02673843.2019.1596823

Orellana López, D. M. y Sánchez Gómez, M. C. (2006). Técnicas de recolección de datos en entornos virtuales más usadas en la investigación cualitativa. Revista de Investigación Educativa, 24(1), 205222. https://revistas.um.es/rie/article/view/97661/93701

Reeves, J. J., Hollandsworth, H. M., Torriani, F. J., Taplitz, R., Abeles, S., Tai-Seale, M. M., Clay, B. J. y Longhurst, C. A. (2020). Rapid Response to COVID-19: Health Informatics Support for Outbreak Management in an Academic Health System. Journal of the American Medical Informatics Association, AMIA, 27(6), 853-859. https://doi.org/10.1093/jamia/ocaa037

Reyes Carmona, C., Monterrosas Rojas, A. M., Navarrete Martinez, A., Acosta Martínez, E. P. y Torruco García, U. (2017). Ansiedad de los estudiantes de una facultad de medicina mexicana, antes de iniciar el internado. Investigación en Educación Médica, 6(21), 42-46. http://www.scielo.org.mx/pdf/iem/v6n21/2007-5057-iem-6-21-00042.pdf

Ries, F., Castañeda Vázquez, C., Campos Mesa, M. C. y Del Castillo, A. O. (2012). Relaciones entre ansiedad-rasgo y ansiedad-estado en competiciones deportivas. Cuadernos de Psicología del Deporte, 12(2), 9-16. http://scielo.isciii.es/pdf/cpd/v12n2/articulo01.pdf 
Sierra, J. C., Ortega, V. y Zubeidat, I. (2003). Ansiedad, angustia y estrés: tres conceptos a diferenciar. Revista Mal-estar E Subjetividade , 3 (1), 10-59. https://www.redalyc.org/pdf/271/27130102.pdf

Singh, I. y Jha, A. (2013). Anxiety, Optimism and Academic Achievement among Students of Private Medical and Engineering Colleges: A Comparative Study. Journal of Educational and Developmental Psychology, 3(1), 222-233. http://dx.doi.org/10.5539/jedp.v3n1p222

Spielberg, C. D. (1993). State-Trait Anxiety Inventory : A CBibliography (2nd ed.). Consulting Psychologists Press. Palo Alto, California, USA.

Thamman, R., Gulati, M., Narang, A., Utengen, A., Mamas, A. M. y Bhatt, D. L. (2020). Twitter-based Learning for Continuing Medical Education? A New Perspective for a Paradigm Shift in Medical Education, Accelerated by COVID-19. European Heart Journal, 1-3. https://doi.org/10.1093/eurheartj/ehaa346

Vitasari, P., Abdul Wahab, M. N., Othman, A., Herawan, T. y Kumar Sinnadurai, S. (2010). The Relationship between Study Anxiety and Academic Performance among Engineering Students. Social and Behavioral Sciences, ELSEVIER, 8, 490-497. https://doi.org/10.1016/j.sbspro.2010.12.067

World Health Organization (WHO). (2020). Archived: WHO Timeline - COVID-19. https://www.who.int/news-room/detail/27-04-2020-who-timeline---covid-19 